\title{
Identification of Apolipoprotein E Guangzhou (Arginine 150 Proline), a New Variant Associated with Lipoprotein Glomerulopathy
}

\author{
Boxun Luo ${ }^{\mathrm{a}}$ Fengxian Huang ${ }^{\mathrm{a}}$ Qicai Liu ${ }^{\mathrm{c}}$ Xiaoyan $\mathrm{Li}^{\mathrm{a}}$ Wenfang Chen ${ }^{\mathrm{b}}$ \\ Shu-Feng Zhou ${ }^{d}$ Xueqing $\mathrm{Yu}^{\mathrm{a}}$ \\ Departments of a Nephrology, and ${ }^{\mathrm{b}}$ Pathology, First Affiliated Hospital, Sun Yet-Sen University, and \\ 'Experiment Medical Research Center, Guangzhou Medical College, Guangzhou, PR China; \\ ${ }^{d}$ Division of Chinese Medicine, School of Health Sciences, WHO Collaborating Center for Traditional Medicine, \\ RMIT University, Bundoora, Vic., Australia
}

\section{Key Words}

Lipoprotein glomerulopathy • Apolipoprotein E • Nephrotic syndrome $\cdot$ Gene mutation

\begin{abstract}
Background/Aims: Lipoprotein glomerulopathy (LPG) is a rare disease characterized by thrombus-like substances in markedly dilated glomerular capillaries and elevated plasma levels of apolipoprotein E (apoE). Previous studies have shown that genetic disorders of apoE may contribute to the pathogenesis of LPG, but LPG may not be caused by apoE gene mutations in Chinese patients. This study investigated the association of a new variant of apoE with LPG in a Chinese family. Methods: The apoE gene in a family with 4 LPG patients was sequenced. The polymerase chain reaction product of coding region of apoE exon 4 was cloned into pMD 18-T vector and then sequenced. Results: A novel point mutation in exon 4 of the apoE gene was identified in all 4
\end{abstract}

B.L. and F.H. contributed equally to this work.
(C) 2007 S. Karger AG, Basel

$0250-8095 / 08 / 0282-0347 \$ 24.50 / 0$

Fax +41613061234

E-Mail karger@karger.ch

www.karger.com
Accessible online at:

www.karger.com/ajn
LPG patients and 1 asymptomatic family member. Sequence analysis confirmed a nucleotide $\mathrm{G}$ to $\mathrm{C}$ point mutation in exon 4 (base 308) of the apoE gene in all patients and the asymptomatic family member. This missense mutation denotes amino acid substitution of the proline residue for arginine residue at position 150 of apoE. Those patients were all heterozygotes with apoE Guangzhou. One of 2 grandsons was a heterozygous carrier of apoE Guangzhou, although he did not have proteinuria. Conclusion: The results of this study suggest that apoE (arginine 150 proline) is a novel apoE variant that etiologically related to LPG. This variant (apoE Guangzhou) may cause a marked molecular conformational change of the apoE and thus impair its binding ability to lipids.

Copyright $\odot 2007$ S. Karger AG, Basel

Lipoprotein glomerulopathy (LPG) is a rare and unique renal disease characterized by the presence of intraglomerular lipoprotein thrombi due to deposition of lipid droplets within the lumina of dilated glomerular capillaries, variable degree of mesangial proliferation, dysbetalipoproteinemia that resembles type III hyperli-

Prof. Xueqing Yu, MD, PhD

Department of Nephrology, The First Affiliated Hospital, Sun Yet-Sen University 58th Zhongshan Road II

Guangzhou 510080 (PR China)

Tel. +86 208776 6335, Fax +8620877 6973, E-Mail yuxq@mail.sysu.edu.cn 
poproteinemia, and elevated levels of serum apolipoprotein $\mathrm{E}$ (apoE) [1-3]. The common clinical presentation of LPG includes proteinuria or nephrotic syndrome, together with moderate renal impairment, which ultimately progresses to chronic renal failure. LPG was first described by Saito et al. [1] in Japanese in 1989 and more than 50 patients have been reported since then [4]. Genetic analysis in these patients indicates that mutations of the $a p o E$ gene increase the risk of LPG and are considered to play an etiological role in LPG [4]. The ApoE gene is located at 19q13.2, with four exons and three introns [5]. ApoE is a major constituent of plasma lipoproteins that is composed of 299 amino acids with molecular weight of $34 \mathrm{kDa}$. It is an important lipoprotein that binds various lipids with many biological actions of great significance [6]. In humans, three common alleles exist that are designated $\varepsilon 2, \varepsilon 3$, and $\varepsilon 4$; their products designated E2, E3, and E4, respectively. The three apoE isoforms differ at polymorphic sites, arginine or cysteine at codons 112 and 158, respectively. ApoE3 and apoE4 appear to bind equally well to lipoprotein receptors, whereas apo E2 (Arg158Cys) has only $\sim 1 \%$ of the binding activity of apoE3 or apoE4 [7]. To date, 54 single nucleotide polymorphisms of the apoE gene have been identified in humans (http://www.ncbi.nlm.nih.gov/sites/entrez). ApoE polymorphisms have a major role in the pathogenesis and progression of a variety of renal diseases: they regulate mesangial and glomerular functions locally in the kidney microenvironment; they are major determinants of plasma lipid levels in uremic patients; they affect the risk for cardiovascular disease in these patients; they predispose to the development of diabetic nephropathy; they affect the severity of certain glomerulopathies; they influence the therapeutic response of some drugs [6].

Variants of apoE including apoE2 (arginine 145 proline) Sendai [8], apoE2 (arginine 25 cysteine) Kyoto [9], and apoE2 (arginine 147 proline) Chicago [4] that are caused by missense mutations, are associated with LPG. On the other hand, variants of $A p o E 1$ with glutamine 156 to glycine $173 \mathrm{del}$ [10], histidine 141 to leucine 144 del or arginine 142 to arginine 146 del Tokyo [11], or histidine 141 to lysine 146 del [12] contain in-frame deletions that confers susceptibility to LPG. All these studies have suggested that LPG may be a dominant inherited disease with incomplete penetrance $[9,10,12]$. However, Chen et al. [13] recently compared the 5.5-kb genomic DNA encompassing the apoE locus and adjoining flanking regions in 17 Chinese LPG patients with 10 normal controls. The DNA sequence analysis did not identify any
apoE gene mutations in all LPG patients, while only 10 variation sites in the non-coding regions were identified [13]. This study suggests that LPG may not be caused by $a p o E$ gene mutations in Chinese patients. Thus, further studies are needed to examine the role of apoE mutations in the pathogenesis of LPG. We reported here on a novel variant of apoE in a Chinese family with 4 LPG patients and 1 asymptomatic family member.

\section{Methods}

\section{Family and Clinical History}

We enrolled 11 members of a family, 4 of whom had histologically confirmed LPG (fig. 1). We performed renal biopsy in the 4 symptomatic family members and diagnosed all of them as having LPG. The mother (72 years old), her daughter (51 years old), her son (49 years old), and 1 of her granddaughters ( 22 years old) had moderate to severe proteinuria (up to $6.0 \mathrm{~g} /$ day) that was accompanied by bipedal edema in 3 patients (all females) and moderate hyperlipidemia in all LPG patients. Other family members were asymptomatic. In LPG patients, the total cholesterol (TC), low-density lipoprotein cholesterol (LDL-C), highdensity lipoprotein cholesterol (HDL-C) and triglyceride (TG) were $328 \mathrm{mg} / \mathrm{dl}(8.15 \mathrm{mmol} / \mathrm{l}), 215 \mathrm{mg} / \mathrm{dl}(5.55 \mathrm{mmol} / \mathrm{l}), 86 \mathrm{mg} /$ $\mathrm{dl}(2.23 \mathrm{mmol} / \mathrm{l})$, and $166 \mathrm{mg} / \mathrm{dl}(1.87 \mathrm{mmol} / \mathrm{l})$, respectively. The clinical symptoms were first noted in the mother at age 62 , in her daughter and son at age 40 , and in her granddaughter at age 12. Serology tests for antinuclear antibodies, anti-DNA antibody, rheumatoid factor, hepatitis B surface antigen, hepatitis C antibody, antiribonuclear protein, anti-Smith, anti-SSA, and anti-SSB were all negative. Blood pressure was in the normal range for all family members. All symptomatic family members were treated with immunosuppressive agents, including prednisone, endoxam, cyclosporine or mycophenolate mofetil, but were not responsive. The mother and her daughter developed progressive renal injury, and had serum creatinine levels up to $7.6 \mathrm{mg} / \mathrm{dl}(676 \mu \mathrm{mol} / \mathrm{l})$. Both of them received kidney transplantation.

\section{Characterization of Plasma Lipids and Lipoproteins}

After obtaining ethics approval and informed consent, we collected fasting blood plasma from all family members. TC and TC levels were measured using a GOD-RAP method (Human Gesellschaft für Biochemica und Diagnostica mbH, Wiesbaden, Germany). The serum levels of LDL-C HDL-C were determined by a homogeneous method (Daiichi Pure Chemicals Co., Tokyo, Japan). The concentrations of plasma apoE, apoA1, and apoB were determined by a single radial immunodiffusion method (Daiichi Pure Chemicals Co.). Our control group consisted of 100 unrelated healthy Chinese individuals, 48 men aged $39 \pm 21$ years and 52 women aged $35 \pm 25$ years (mean $\pm \mathrm{SD}$ ).

Renal Pathology

Formalin-fixed, 4- $\mu \mathrm{m}$ paraffin sections from renal biopsy tissues were treated with hematoxylin and eosin, periodic acidSchiff (PAS), and Masson trichrome staining. Glomerulosclerosis was indicated by the presence of a large amount of PAS and tri- 
Fig. 1. Light microscopy $(200 \times)$ of a glomerulus in the biopsy specimen of a symptomatic LPG patient (II-1, proband). The capillary lumina were dilated by amorphous thrombi. a HE stain; b Masson stain; c PAS stain; d PASM stain.

Fig. 2. Nucleotide sequence of a segment of exon 4 in the apoE gene from both alleles of the proband (patient II-1). The lower panel shows the allele containing a nucleotide $\mathrm{G}$ to $\mathrm{C}$ point mutation at nucleotide 308 in codon 150 of the gene.

New apoE Variant in Lipoprotein Glomerulopathy
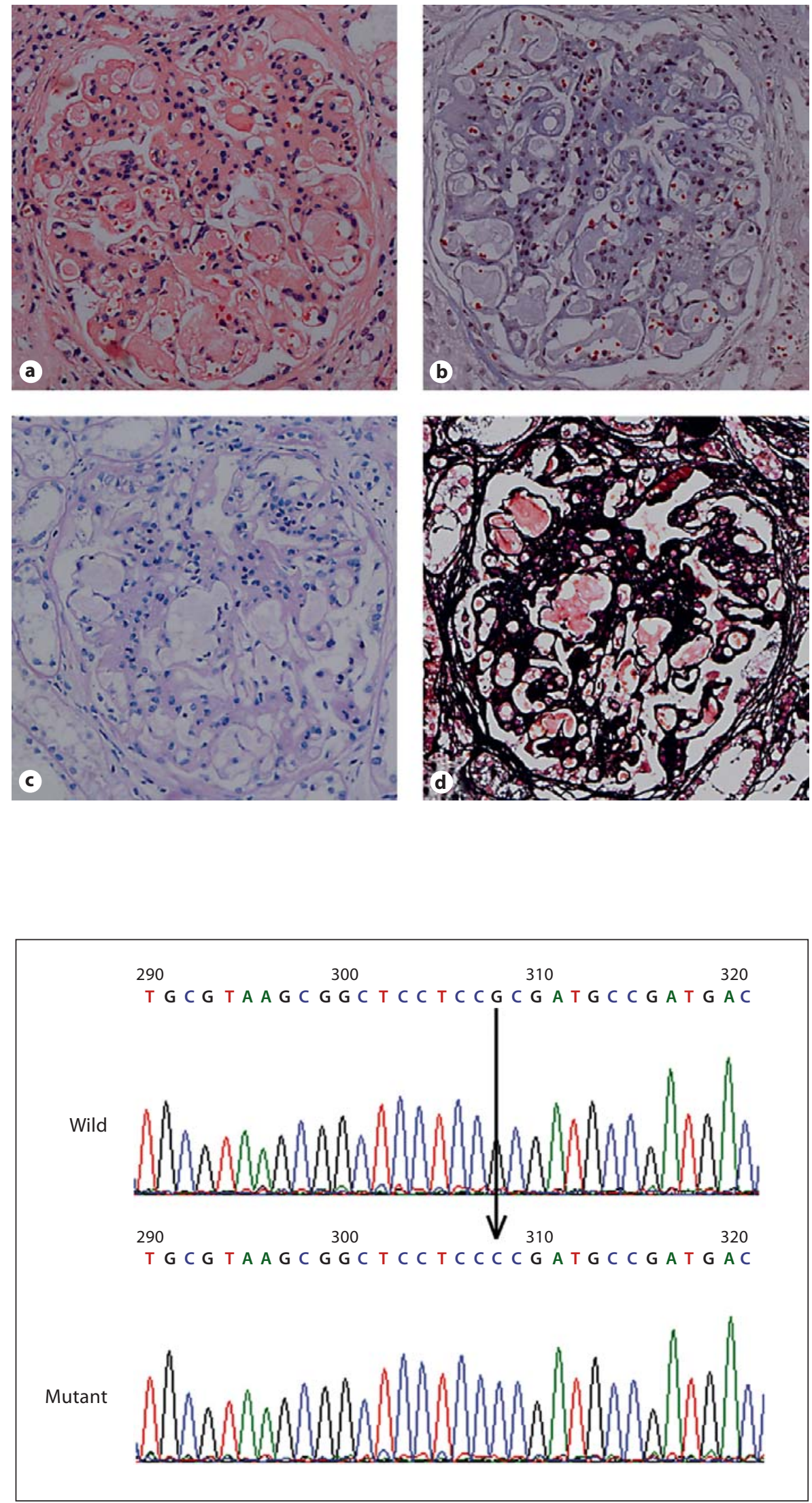

Am J Nephrol 2008;28:347-353 
chrome-positive materials within the expanded mesangium. Tubulointerstitial fibrosis was demonstrated by fibroblast and extracellular matrix accumulation, accompanied by a loss or atrophy of tubules.

DNA Amplification by Polymerase Chain Reaction (PCR)

Genomic DNA was extracted from patients' blood using the DNA Blood MaxiPrep Kit (Qiagen, Inc., Valencia, Calif., USA) and used $1 \mathrm{ml}$ of the extraction as a template for PCR amplification. The fragment of the apoE gene covering exon 4 was amplified by using PCR SuperMix High Fidelity (Invitrogen, Carlsbad, Calif., USA). The primers for exon 4 were: forward 5'-AGGGCGCTGATGGACGAG-3' and reverse 5'-GCCAGCAGATGCGTGAAACTT-3'. Typical amplification reactions of $50 \mu l$ contained $100 \mathrm{ng}$ genomic DNA and $200 \mathrm{nmol} / \mathrm{l}$ of oligonucleotide primers. The DNA was denatured at $94^{\circ} \mathrm{C}$ for $30 \mathrm{~s}$, annealed at $58^{\circ} \mathrm{C}$ for $30 \mathrm{~s}$, and extended at $72^{\circ} \mathrm{C}$ for $45 \mathrm{~s}$ for a total of 33 cycles using a thermocycler (TGradient, Biometra, Germany). The access number for human apoE gene in GenBank is NM_000041.

\section{DNA Sequence Analysis}

The resultant PCR products were purified with the QIAquick Gel Extraction kit (Qiagen, Inc.) and ligated the gel-purified PCR products into a pMD 18 -T vector (TaKaRa). All 12 clones were sequenced using an ABI 3730 automated DNA sequencer (Applied Biosystems, Foster City, Calif., USA).

\section{Statistical Analysis}

Data are expressed as mean \pm SD. Statistical significances were evaluated using unpaired Student's t tests. Statistical analyses were performed using SAS 8.0 (SAS Institute Inc., Cary, N.C., USA) and the level of statistical significance was set at $\mathrm{p}<$ 0.05 .

\section{Results}

\section{Pathological Changes}

Figure 1 shows the abnormal glomerular architecture of an LPG patient. The glomerular structure was damaged by widespread amorphous glomerular capillary thrombi that were PAS-negative and trichrome-negative. The glomeruli were enlarged with mesangial sclerosis and hypercellularity and there was remarkable focal interstitial fibrosis and tubular atrophy.

\section{DNA Sequence Analysis}

We analyzed 12 independent clones of exon 4 of the $a p o E$ gene by DNA sequencing. The sequences of 5 clones were identical to the normal apoE3 allele, whereas 7 clones had a nucleotide substitution of $\mathrm{G}$ to $\mathrm{C}$ at codon 150 (base 308) on exon 4 of the $a p o E$ gene. Figure 2 shows the nucleotide sequence of part of exon 4 of both alleles of the proband II-1. This missense mutation is a new $a p o E$ variant, with amino acid substitution of arginine 150

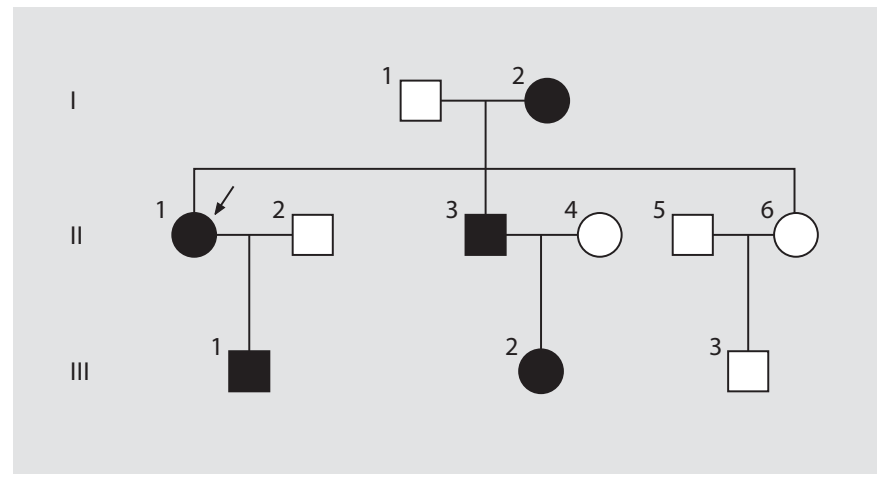

Fig. 3. Family tree of patients with LPG. The proband (II-1) is indicated by the arrow. Square represents male, circle represents female, blank symbols represent unaffected family members, and black symbols represent patients heterozygous for the apolipoprotein E Guangzhou (arginine 150 proline). III-1 is an asymptomatic carrier of the mutant.

proline. There were no other sequence abnormalities identified in our study patients. In addition, none of the healthy control individuals contained the apoE (arginine 150 proline) variant.

\section{Familiar Study}

The pedigree of the patient is illustrated in figure 3. The mother (I-2), her daughter (II-1), her son (II-3), 1 of her grandsons (III-1), and a granddaughter (III-2) were heterozygous carriers of the same mutation (arginine 150 proline). Carriers I-2, II-1, II-3, and II-2 presented significant clinical symptoms of renal injuries. However, the carrier (III-1) did not show any clinical symptoms or signs of renal damage.

\section{Lipid and Lipoprotein Profiles}

Table 1 summarizes the plasma lipid and lipoprotein profiles of carrier and non-carrier of the family. Carriers of the new apoE mutation had significantly higher levels of plasma TG, TC, HDL-C and apoE but a lower LDL-C level compare with non-carriers of the new apoE mutation or healthy subjects $(\mathrm{p}<0.05)$, although patients I-2, II-1, II-3, III-2 were taking lipid-lowering agents when we performed this analysis. In particular, the plasma apoE level was almost doubled. There was no significant difference in the plasma levels of apoA 1 and apoB between carriers and non-carriers of the apoE mutation, but the carriers had a significantly lower apoB level compared to healthy subjects. Notably, the non-carriers had significantly higher TC level but a higher level of LDL-C com- 
Table 1. Plasma lipid and lipoprotein profiles of family members with and without LPG

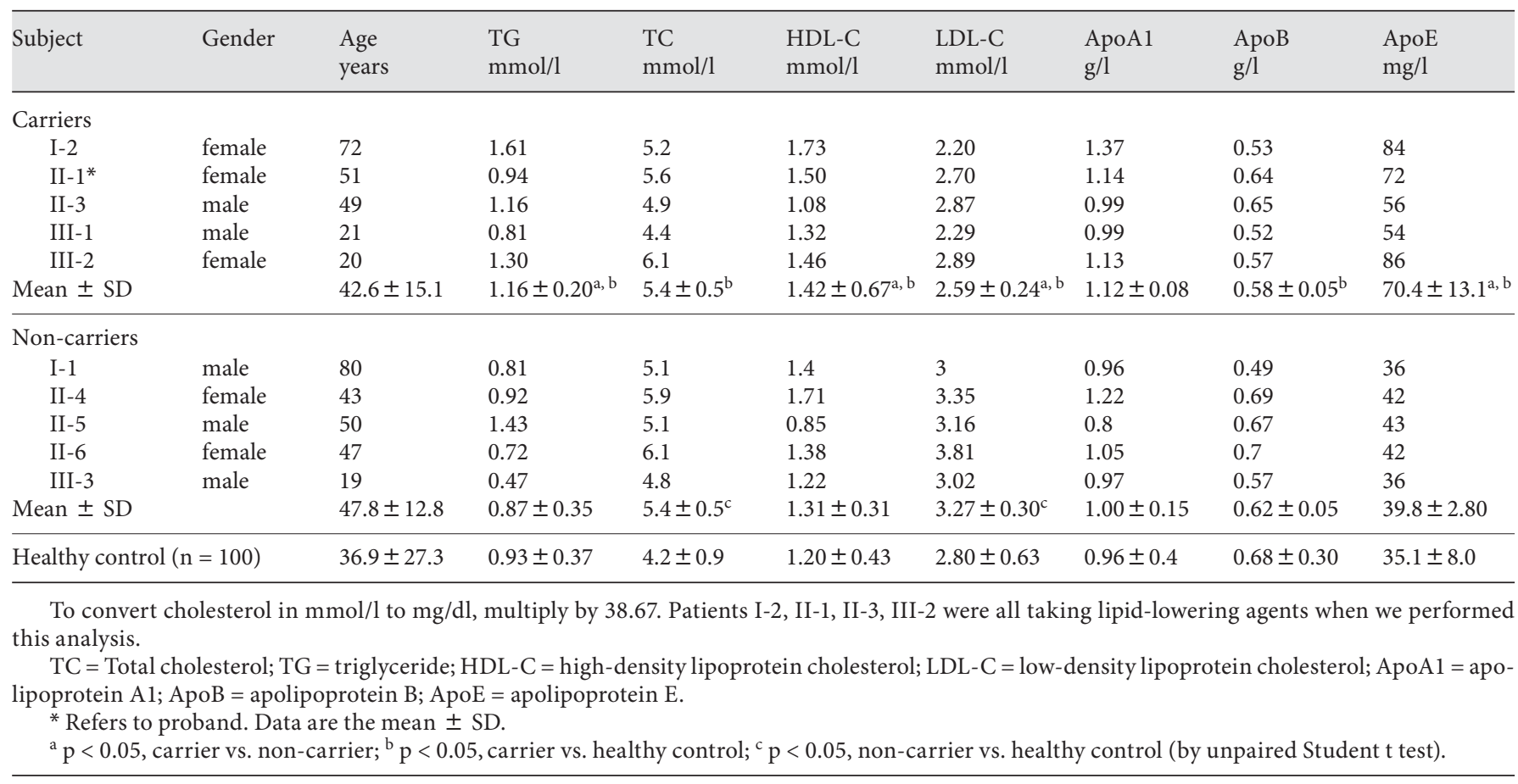

pared with healthy subjects, suggesting that the family members of carriers may have abnormal lipid metabolism.

\section{Discussion}

The etiology of LPG is uncertain, but many researchers believe that mutation of the $a p o E$ gene may play an important role. Thus, detailed characterization of $a p o E$ genotypes and their functional impact is crucial to explore the pathogenesis of LPG. It has been reported that various mutations of the apoE gene were present in patients with LPG [4, 8-12]. In addition, Ishigaki et al. [14] established an animal model of LPG by introducing a recombinant adenovirus containing human apoE-Sendai into apoE knockout mice. In the present study, we identified a novel variant of apoE in a Chinese family with 4 LPG patients and 1 asymptomatic family member (fig. 3). We provisionally named the variant 'apoE Guangzhou' after our city. This is the first report in a family with LPG in three generations. Our study suggests that the presence of a mutant apoE Guangzhou (arginine 150 proline, $308 \mathrm{G}>\mathrm{C}$ ) is etiologically related to LPG. This is in contrary to the findings by Chen at al. [13] in which no non- synonymous mutations were detected in 17 Chinese LPG patients.

Histological examination confirmed the diagnosis of LPG in the Chinese family. The glomerular injuries seen in our patients are characteristic of LPG as reported previously [4]. Under the microscope, there were amorphous glomerular capillary thrombi that were PAS-negative and trichrome-negative (fig. 1). Marked focal interstitial fibrosis and tubular atrophy were observed and the glomeruli were filled with mesangial sclerosis and hypercellularity. The abnormal lipid profiles of these LPG patients in our study were characterized by increased plasma levels of TC, TG, HDL-C and apoE (table 1). It would be interesting to investigate the phenotype of apoE and the binding affinity of the mutant apoE to LDL receptor in these LPG patients.

The major physiological role of apoE is mediation of tissue uptake of TC-rich lipoproteins through the LDL receptor and the LDL receptor-related protein pathways [15]. Human apoE is a glycoprotein composed of 299 amino acids, with residues 136-150 constituting the region that is responsible for binding to the LDL receptor [16]. In the present report, apoE (arginine 150 proline) Guangzhou had a substitution of proline for arginine at residue 150 . Since prolines are helix breakers in globular 
proteins [15], we speculate that the substitution of proline for arginine may change the protein structure. The crucial molecular transformation in the apoE Guangzhou appears to lead to aggregated deposits of lipoproteins that have high affinity or low clearance in glomerulus. Interestingly, apoE2 (arginine 145 proline) Sendai, apoE2 (arginine 147 proline) Chicago, and apoE (arginine150 proline) Guangzhou all have a guanine to cytosine point mutation in exon 4 of the apoE gene at a position that is in the LDL receptor-binding site. Previous research has shown that mutant apoEs are defective in binding to the LDL receptor, but exhibit enhanced binding to the glomerulus $[4,9,14,17]$, contributing to the development of LPG. Since apoE mRNA is expressed in normal kidney [18], upregulation of apoE in glomeruli may also account for the development of LPG [19]. However, the recurrence of LPG after renal transplantation [20-23] suggests that apoE deposition within the glomerulus may derive from circulation, not from local synthesis in the glomerulus.

Recent studies show that the mutation of $a p o E$ is transmitted as autosomal dominant with incomplete pene- trance $[9,10,12,21]$. This may explain our finding that 1 carrier of the apoE (arginine 150 proline) mutation had normal renal function, despite having an increased abnormal serum apoE level $(54 \mathrm{mg} / \mathrm{l})$. However, a long-term follow-up is required for this carrier because the asymptomatic carrier was only 21 years old. It is unlikely to rule out early glomerular injury since we did not perform renal biopsy on this patient. A previous study reported that lipoprotein glomerular damage can occur earlier than any clinical manifestations [23].

In summary, we have identified a new variant of apoE, apoE Guangzhou, in 3 generations of a family which included 4 LPG patients and 1 asymptomatic carrier. Further study of the characteristics of this variant will provide insights into the pathogenesis of LPG.

\section{Acknowledgments}

We thank Prof. Huiyao Lan (The University of Hong Kong, Li Ka Shing Faculty of Medicine) and Prof. Guanqing Wu (Vanderbilt University School of Medicine) for their discussions and comments.

\section{References}

1 Saito T, Sato H, Kudo K, Oikawa S, Shibata T, Hara Y, Yoshinaga K, Sakaguchi H: Lipoprotein glomerulopathy: glomerular lipoprotein thrombi in a patient with hyperlipoproteinemia. Am J Kidney Dis 1989;13. 148-153.

- Watanabe Y, Ozaki I, Yoshida F, Fukatsu A, Itoh Y, Matsuo S, Sakamoto N: A case of nephrotic syndrome with glomerular lipoprotein deposition with capillary ballooning and mesangiolysis. Nephron 1989;51:265270.

3 Saito T, Sato H, Oikawa S, Kudo K, Kurihara I, Nakayama K, Abe K, Yoshinaga K, Sakaguchi $\mathrm{H}$ : Lipoprotein glomerulopathy. Report of a normolipidemic case and review of the literature. Am J Nephrol 1993;13:64-68.

-4 Sam R, Wu H, Yue L, Mazzone T, Schwartz MM, Arruda JA, Dunea G, Singh AK: Lipoprotein glomerulopathy: a new apolipoprotein E mutation with enhanced glomerular binding. Am J Kidney Dis 2006;47:539-548.

5 Shaw DJ, Brook JD, Meredith AL, Harley HG, Sarfarazi M, Harper PS: Gene mapping and chromosome 19. J Med Genet 1986;23: 2-10.

6 Liberopoulos E, Siamopoulos K, Elisaf M: Apolipoprotein E and renal disease. Am J Kidney Dis 2004;43:223-233.
Marcel YL, Kiss RS: Structure-function relationships of apolipoprotein A-I: a flexible protein with dynamic lipid associations. Curr Opin Lipidol 2003;14:151-157.

8 Oikawa S, Matsunaga A, Saito T, Sato H, Seki T, Hoshi K, Hayasaka K, Kotake H, Midorikawa H, Sekikawa A, Hara S, Abe K, Toyota T, Jingami H, Nakamura H, Sasaki J: Apolipoprotein E Sendai (arginine $145 \rightarrow$ proline): a new variant associated with lipoprotein glomerulopathy. J Am Soc Nephrol 1997;8: 820-823.

-9 Matsunaga A, Sasaki J, Komatsu T, Kanatsu K, Tsuji E, Moriyama K, Koga T, Arakawa K, Oikawa S, Saito T, Kita T, Doi T: A novel apolipoprotein E mutation, E2 (Arg25Cys), in lipoprotein glomerulopathy. Kidney Int 1999;56:421-427.

10 Ando M, Sasaki J, Hua H, Matsunaga A, Uchida K, Jou K, Oikawa S, Saito T, Nihei H: A novel 18-amino-acid deletion in apolipoprotein E associated with lipoprotein glomerulopathy. Kidney Int 1999;56:13171323.

11 Konishi K, Saruta T, Kuramochi S, Oikawa S, Saito T, Han H, Matsunaga A, Sasaki J: Association of a novel 3-amino-acid deletion mutation of apolipoprotein E (Apo E Tokyo) with lipoprotein glomerulopathy. Nephron 1999;83:214-218.
12 Ogawa T, Maruyama K, Hattori H, Arai H, Kondoh I, Egashira T, Watanabe T, Kobayashi Y, Morikawa A: A new variant of apolipoprotein E (apo E Maebashi) in lipoprotein glomerulopathy. Pediatr Nephrol 2000;14: 149-151.

13 Chen S, Liu ZH, Zheng JM, Zhang X, Li LS: A complete genomic analysis of the apolipoprotein E gene in Chinese patients with lipoprotein glomerulopathy. J Nephrol 2007;20: 568-575.

14 Ishigaki Y, Oikawa S, Suzuki T, Usui S, Magoori K, Kim DH, Suzuki H, Sasaki J, Sasano H, Okazaki M, Toyota T, Saito T, Yamamoto TT: Virus-mediated transduction of apolipoprotein E (ApoE)-Sendai develops lipoprotein glomerulopathy in ApoE-deficient mice. J Biol Chem 2000;275:31269-31273.

15 Mahley RW: Apolipoprotein E: cholesterol transport protein with expanding role in cell biology. Science 1988;240:622-630.

16 Lalazar A, Weisgraber KH, Rall SC Jr, Giladi $\mathrm{H}$, Innerarity TL, Levanon AZ, Boyles JK, Amit B, Gorecki M, Mahley RW, et al: Sitespecific mutagenesis of human apolipoprotein E. Receptor binding activity of variants with single amino acid substitutions. J Biol Chem 1988;263:3542-3545. 
17 Hoffmann M, Scharnagl H, Panagiotou E, Banghard W, Wieland H, Marz W: Diminished LDL receptor and high heparin binding of apolipoprotein E2 Sendai associated with lipoprotein glomerulopathy. J Am Soc Nephrol 2001;12:524-530.

18 Elshourbagy NA, Liao WS, Mahley RW, Taylor JM: Apolipoprotein E mRNA is abundant in the brain and adrenals, as well as in the liver, and is present in other peripheral tissues of rats and marmosets. Proc Natl Acad Sci USA 1985;82:203-207.
19 Saito T, Oikawa S, Sato H, Sato T, Ito S, Sasaki J: Lipoprotein glomerulopathy: significance of lipoprotein and ultrastructural features. Kidney Int suppl 1999;71:S37-S41.

20 Djamali A, Cristol JP, Turc-Baron C, Beucler I, Baldet P, Mourad G: Lipoprotein glomerulopathy: a new French case with recurrence on the transplant (in French). Presse Med 1996;25:798-802.

21 Mourad G, Cristol JP, Turc-Baron C, Djamali A: Lipoprotein glomerulopathy: a new apolipoprotein-E-related disease that recurs after renal transplantation. Transplant Proc 1997;29:2376.
22 Andrews PA, O’Donnell PJ, Dilly SA, Snowden SA, Bewick M: Recurrence of lipoprotein glomerulopathy after renal transplantation. Nephrol Dial Transplant 1997; 12:2442-2444.

23 Miyata T, Sugiyama S, Nangaku M, Suzuki D, Uragami K, Inagi R, Sakai H, Kurokawa $\mathrm{K}$ : Apolipoprotein E2/E5 variants in lipoprotein glomerulopathy recurred in transplanted kidney. J Am Soc Nephrol 1999;10: 1590-1595. 